\title{
RECTANGULAR HEXAHEDRONS AS FERMAT BASES OF QUADRICS
}

\author{
ZVONKO ČERIN
}

\begin{abstract}
The concepts of a Fermat base of a quadric and of a Fermat locus of a rectangular hexahedron come from an old geometric problem by Pierre de Fermat about a semicircle on a side of a rectangle with ratio of adjacent sides equal to $\sqrt{2}$, which was resolved by synthetic methods first by Leonard Euler in 1750. An arbitrary rectangular hexahedron has a quadric as its Fermat locus. This quadric is either an ellipsoid, a rotational paraboloid or a hyperboloid with two sheets. Conversely, for every quadric from any of these three types one can ask to find all of its rectangular hexahedron Fermat bases which share a line of symmetry with the quadric.
\end{abstract}

\section{INTRODUCTION}

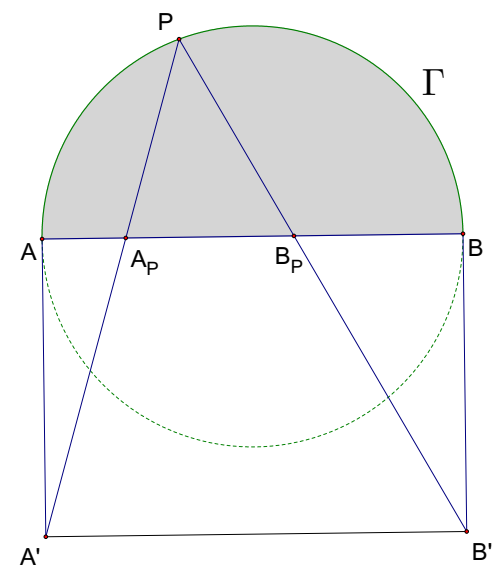

Figure 1: The configuration of the Fermat problem

2010 Mathematics Subject Classification. Primary 54H01.

Key words and phrases. Fermat (geometric) problem, semicircle, rectangular hexahedron, quadric, sphere, ellipsoid, rotational paraboloid, hyperboloid with two sheets. 
For a rectangle $R=A B B^{\prime} A^{\prime}$ in the plane, let us call $s(R)=\frac{|A B|}{\left|A A^{\prime}\right|}$ its shape. Among the numerous questions that Pierre de Fermat has formulated, the following geometric problem is our main concern (see Fig. 1).

Fermat Problem. Let $R=A B B^{\prime} A^{\prime}$ be a rectangle with the shape $\sqrt{2}$ and let $\Gamma$ denote the semicircle on the side $A B$ as a diameter. Prove that for every point $P \in \Gamma$ the relation $\left|A B_{P}\right|^{2}+\left|B A_{P}\right|^{2}=|A B|^{2}$ holds, where $A_{P}$ and $B_{P}$ are intersections of the line $A B$ with the lines $A^{\prime} P$ and $B^{\prime} P$.

The great Leonard Euler in [7] has provided the first rather long proof, which is old fashioned (for his time), and avoids the analytic geometry (which offers rather simple proofs). Several more concise synthetic proofs are now known (see [11], [8, pp. 602, 603], [1, pp. 168, 169] and [9, pp. 181, 264]).

The author and G. M. Gianella have done some recent contributions in [2], [3], [4], [5] and [6]. See also a nice description of Euler's proof in [12].

In this paper we consider the space version of the Fermat problem for quadrics extending our results in [5] which considers only spheres.

Let $v$ be a positive real number. For a rectangular hexahedron $H=$ $A B C D A^{\prime} B^{\prime} C^{\prime} D^{\prime}$ and a surface $\Gamma$ in the 3 -dimensional Euclidean space, we use the notation $H \stackrel{v}{\simeq} \Gamma$ provided for every point $P$ on $\Gamma$ the relation

$$
\left|A C_{P}\right|^{2}+\left|B D_{P}\right|^{2}+\left|C A_{P}\right|^{2}+\left|D B_{P}\right|^{2}=v\left(|A C|^{2}+|B D|^{2}\right)
$$

holds, where $A_{P}, B_{P}, C_{P}$ and $D_{P}$ are the intersections of the plane $\pi_{T}$ determined by the top face $T=A B C D$ of $H$ with the lines $A^{\prime} P, B^{\prime} P, C^{\prime} P$ and $D^{\prime} P$ (see Fig. 2). In this situation, we say that $H$ is an $F^{v}$-base for $\Gamma$ or that $\Gamma$ is an $F^{v}$-locus of $H$ (since $\Gamma$ is generated from $H$ ).

Our idea is to put the rectangular hexahedron $H=A B C D A^{\prime} B^{\prime} C^{\prime} D^{\prime}$ at the center of the stage and try to determine the surface $\Gamma$ such that the relation $H \stackrel{v}{\simeq} \Gamma$ holds. Using analytic geometry, it is easy to show that $\Gamma$ is a quadric (i. e., a surface of order two). The type of this quadric depends on the number $v$ and the shape $s=\frac{|A C|}{\left|A A^{\prime}\right|}$ of the rectangle $A C C^{\prime} A^{\prime}$. Only three types are possible: an ellipsoid for $0<v<2$, a rotational paraboloid for $v=2$ and a hyperboloid with two sheets for $v>2$ and $\frac{2}{\sqrt{v-2}}>s>\frac{1}{\sqrt{v-2}}$.

In the rest of this paper, we search for conditions when will a rectangular hexahedron be an $F^{v}$-base of a given quadric. It turns out that the cases of a rotational paraboloid, a hyperboloid with two sheets, an ellipsoid and a sphere each have their own peculiarities so that we treat them separately. In each of these results the surface and its rectangular hexahedron $F^{v}$-base share a line of symmetry. 


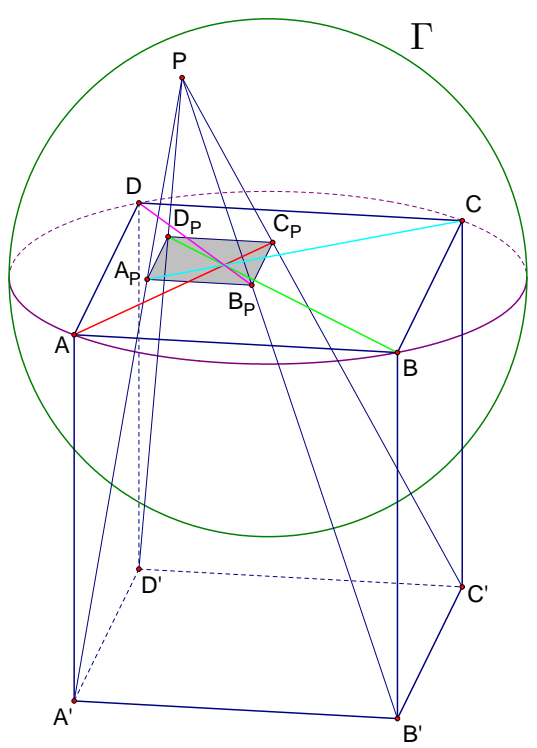

Figure 2: The space version of the Fermat problem

\section{2. $F^{v}$-LOCI OF RECTANGULAR HEXAHEDRONS}

Theorem 1. Let $v$ be a positive real number. Every rectangular hexahedron $H=A B C D A^{\prime} B^{\prime} C^{\prime} D^{\prime}$ is an $F^{v}$-base of some quadric $\Gamma$. This quadric is an ellipsoid for $v<2$, a rotational paraboloid for $v=2$ and a hyperboloid with two sheets when $v>2$ and $\frac{2}{\sqrt{v-2}}>s>\frac{1}{\sqrt{v-2}}$, where $s=s\left(A C C^{\prime} A^{\prime}\right)=\frac{|A C|}{\left|A A^{\prime}\right|}$ is the shape of the rectangle $A C C^{\prime} A^{\prime}$.

Proof. We shall use analytic geometry, which offers a simple proof. Let the origin of the rectangular coordinate system in the 3-dimensional Euclidean space be the midpoint $O$ of the diagonal $A C$ of the top face $T=A B C D$ so that the points $A, B, C$ and $D$ have coordinates $(-a, b, 0),(a, b, 0),(a,-b, 0)$ and $(-a,-b, 0)$ for some positive real numbers $a$ and $b$. The coordinates of the points $A^{\prime}, B^{\prime}, C^{\prime}$ and $D^{\prime}$ are $(-a, b, m),(a, b, m),(a,-b, m)$ and $(-a,-b, m)$ for some real number $m \neq 0$.

An arbitrary point $P$ on the required locus has coordinates $(x, y, z)$. From the similar right-angled triangles, we find $A_{P}\left(\frac{m x+a z}{m-z}, \frac{m y-b z}{m-z}, 0\right)$, $B_{P}\left(\frac{m x-a z}{m-z}, \frac{m y-b z}{m-z}, 0\right), C_{P}\left(\frac{m x-a z}{m-z}, \frac{m y+b z}{z-m}, 0\right)$ and $D_{P}\left(\frac{m x+a z}{m-z}, \frac{m y+b z}{z-m}, 0\right)$. Then $\left|A C_{P}\right|^{2}+\left|B D_{P}\right|^{2}+\left|C A_{P}\right|^{2}+\left|D B_{P}\right|^{2}=v\left(|A C|^{2}+|B D|^{2}\right)$ holds if and 
only if $m^{2}\left(x^{2}+y^{2}\right)=c^{2}\left[2(m-z)^{2} v-(m-2 z)^{2}\right]$, where $c$ is $\sqrt{a^{2}+b^{2}}$. Hence, the locus is a quadric.

The above equation has the familiar form $\Xi=0$, with $\Xi$ equal to

$$
\begin{aligned}
a_{11} x^{2}+a_{22} y^{2}+a_{33} z^{2}+2 a_{12} x y & +2 a_{13} x z \\
& +2 a_{23} y z+2 a_{14} x+2 a_{24} y+2 a_{34} z+a_{44},
\end{aligned}
$$

with $a_{11}=a_{22}=m^{2}, a_{33}=2 c^{2}(2-v), a_{12}=a_{13}=a_{23}=a_{14}=a_{24}=0$, $a_{34}=2 m c^{2}(v-1)$ and $a_{44}=m^{2} c^{2}(1-2 v)$.

It is well-known (see $[10,3.5]$ ) that the invariants

$$
\begin{gathered}
I=a_{11}+a_{22}+a_{33}=2\left[m^{2}+c^{2}(2-v)\right], \\
J=\left|\begin{array}{ll}
a_{11} & a_{12} \\
a_{12} & a_{22}
\end{array}\right|+\left|\begin{array}{ll}
a_{22} & a_{23} \\
a_{23} & a_{33}
\end{array}\right|+\left|\begin{array}{ll}
a_{33} & a_{13} \\
a_{13} & a_{11}
\end{array}\right|=m^{2}\left[m^{2}+4 c^{2}(2-v)\right], \\
\mathcal{D}=\left|\begin{array}{lll}
a_{11} & a_{12} & a_{13} \\
a_{12} & a_{22} & a_{23} \\
a_{13} & a_{23} & a_{33}
\end{array}\right|=2 m^{4} c^{2}(2-v),
\end{gathered}
$$

and

$$
\mathcal{A}=\left|\begin{array}{llll}
a_{11} & a_{12} & a_{13} & a_{14} \\
a_{12} & a_{22} & a_{23} & a_{24} \\
a_{13} & a_{23} & a_{33} & a_{34} \\
a_{14} & a_{24} & a_{34} & a_{44}
\end{array}\right|=-2 c^{4} m^{6} v
$$

together determine the properties of a quadric which do not depend on its position on the space.

Since $\mathcal{A} \neq 0$, the quadric is non-degenerate. For $v=2$, we have $\mathcal{D}=0$ and $I^{2}=4 J$ so that $\Gamma$ is a rotational paraboloid.

For $0<v<2$, the invariants satisfy $\mathcal{A}<0, \mathcal{D}>0, I>0$ and $J>0$ so that $\Gamma$ is an ellipsoid.

Finally, for $v>2$, we have $\mathcal{A}<0, \mathcal{D} I=4 m^{4} \lambda\left(m^{2}+\lambda\right)$ and $J=m^{2}$ $\left(m^{2}+4 \lambda\right)$, where $\lambda=c^{2}(2-v)$. We must consider the following two cases: (Case I) When is $\mathcal{D} I>0$ and $J>0$ ? and (Case II) When is $\mathcal{D} I<0$ and $J<0$ ?

(Case I). Note that $\mathcal{D} I>0$ holds only for $\lambda>0$ and $\lambda<-m^{2}$. But, $\lambda>0$ is not true for $v>2$. Hence, $\lambda<-m^{2}$. On the other hand, $J>0$ is true only for $\lambda>-\frac{m^{2}}{4}$. These two conditions on $\lambda$ are incompatible so that this case can not happen.

(Case II). The inequality $\mathcal{D} I<0$ holds only for $0>\lambda>-m^{2}$ while the inequality $J<0$ holds only for $\lambda<-\frac{m^{2}}{4}$. Hence, $-m^{2}<\lambda<-\frac{m^{2}}{4}$. Since $s=\frac{2 c}{m}$, we conclude that this case happens if and only if the inequality 
$\frac{2}{\sqrt{v-2}}>s>\frac{1}{\sqrt{v-2}}$ is true. According to Table 3.5-1 in [10], in this case the quadric $\Gamma$ is a hyperboloid with two sheets.

\section{The CASE of a Rotational Paraboloid}

When $v=2$, the Fermat configuration is tied to a rotational paraboloid. This is explained in the following result (see Fig. 3).

Theorem 2. For every rotational paraboloid $\delta$ and every pair $\lambda=(a, b)$ of real numbers $a$ and $b$ both different from zero, there is a rectangular hexahedron $H_{\lambda}=A_{\lambda} B_{\lambda} C_{\lambda} D_{\lambda} A_{\lambda}^{\prime} B_{\lambda}^{\prime} C_{\lambda}^{\prime} D_{\lambda}^{\prime}$ such that $H_{\lambda}$ and $\delta$ have a common line of symmetry and for a point $P$ in the space the following two statements are equivalent:

(a) A point $P$ is on the rotational paraboloid $\delta$.

(b) The sum $\left|A C_{P}\right|^{2}+\left|B D_{P}\right|^{2}+\left|C A_{P}\right|^{2}+\left|D B_{P}\right|^{2}$ is equal to the product $2\left(|A C|^{2}+|B D|^{2}\right)$, where $A_{P}, B_{P}, C_{P}$ and $D_{P}$ are intersections of the plane $A_{\lambda} B_{\lambda} C_{\lambda} D_{\lambda}$ with the lines $A_{\lambda}^{\prime} P, B_{\lambda}^{\prime}, C_{\lambda}^{\prime} P$ and $D_{\lambda}^{\prime} P$.

Proof. Without loss of generality, we can assume that the line of symmetry $\pi$ of $\delta$ is the $z$-axis of the rectangular coordinate system in the 3 -dimensional Euclidean space, the equation of $\delta$ is a standard $x^{2}+y^{2}=2 k z$ for a positive real number $k$ and the coordinates of the vertices $A, B, C, D, A^{\prime}, B^{\prime}, C^{\prime}$ and $D^{\prime}$ of a rectangular hexahedron $A B C D A^{\prime} B^{\prime} C^{\prime} D^{\prime}$ are $(-a, b, n),(a, b, n)$, $(a,-b n),(-a,-b, n),(-a, b, m),(a, b, m),(a,-b m)$ and $(-a,-b, m)$, where $a, b, n$ and $m$ are real numbers such that $m \neq n, a \neq 0$ and $b \neq 0$. An arbitrary point $P$ in the space has coordinates $(x, y, z)$. Let $\alpha=n-m$, $\beta=n-z$ and $\gamma=z-m$. The coordinates of the intersections $A_{P}, B_{P}, C_{P}$ and $D_{P}$ are $\left(\frac{\alpha x+\beta a}{\gamma}, \frac{\alpha y-\beta b}{\gamma}, n\right),\left(\frac{\alpha x-\beta a}{\gamma}, \frac{\alpha y-\beta b}{\gamma}, n\right),\left(\frac{\alpha x-\beta a}{\gamma}, \frac{\alpha y+\beta b}{\gamma}, n\right)$ and $\left(\frac{\alpha x+\beta a}{\gamma}, \frac{\alpha y+\beta b}{\gamma}, n\right)$. Then

$$
\left|A C_{P}\right|^{2}+\left|B D_{P}\right|^{2}+\left|C A_{P}\right|^{2}+\left|D B_{P}\right|^{2}-2\left(|A C|^{2}+|B D|^{2}\right)=\frac{4 \alpha M_{p}}{\gamma^{2}},
$$

where $M_{p}=\alpha^{2}\left(x^{2}+y^{2}\right)+c^{2}(\alpha-4 \gamma)$ and $c^{2}=a^{2}+b^{2}$.

Now, in order that $P$ is on the rotational paraboloid $\delta$, the polynomial $M_{p}$ should be of the form $t\left(x^{2}+y^{2}-2 k z\right)$ for some real number $t \neq 0$. This gives the system of ten equations in variables $a, b, n, m$ and $t$. The only solution is $n=\frac{3 c^{2}}{2 k}, m=-\frac{c^{2}}{2 k}$ and $t=2 c^{2}$ that gives the required rectangular hexahedron $H_{\lambda}$ with vertices $\left(-a, b, n_{0}\right),\left(a, b, n_{0}\right),\left(a,-b, n_{0}\right),\left(-a,-b, n_{0}\right)$, $\left(-a, b, m_{0}\right),\left(a, b, m_{0}\right),\left(a,-b, m_{0}\right)$ and $\left(-a,-b, m_{0}\right)$, where $n_{0}=\frac{3 c^{2}}{2 k}$ and $m_{0}=-\frac{c^{2}}{2 k}$. 


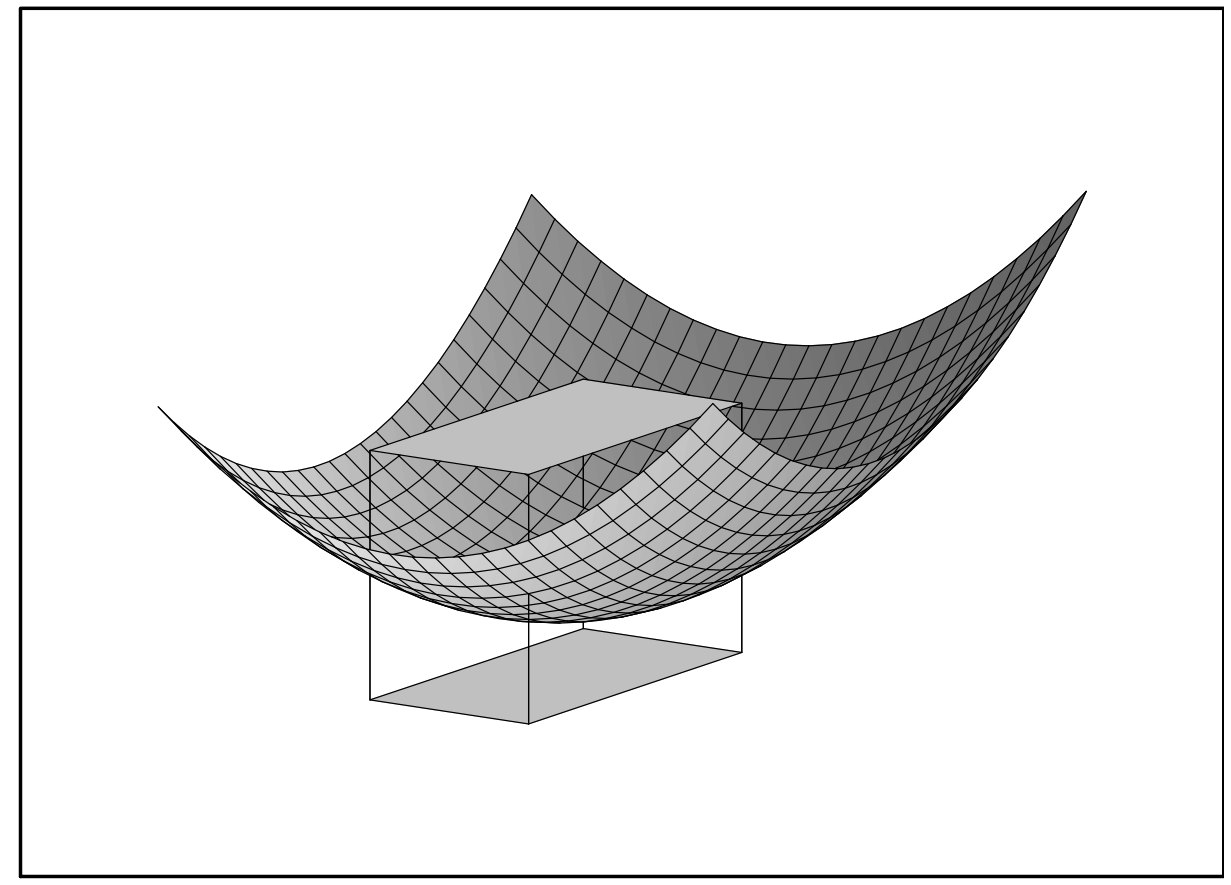

Figure 3: Theorem 2 for $a=1, b=2$ and $k=1$.

\section{The CASE OF AN ELLIPSOID}

When $v<2$, some special ellipsoids have rectangular hexahedrons as their $F^{v}$-bases. This is the content of the following result (see Fig. 4). Note that these rectangular hexahedrons have the $z$-axis as a line of symmetry.

Theorem 3. Let real numbers $b, h, m$ and $v$ satisfy $b>0, h>0, m \neq 0$ and $0<v<2$. Let $\Gamma$ be the ellipsoid $\frac{x^{2}}{h^{2}}+\frac{y^{2}}{h^{2}}+\frac{z^{2}}{2 v m^{2}}=1$. If $b<h \sqrt{\frac{2}{v}-1}$, then there are two rectangular hexahedrons $H=A B C D A^{\prime} B^{\prime} C^{\prime} D^{\prime}$ with vertices $( \pm e, b, d),(\mp e, b, d),(\mp e,-b, d),( \pm e,-b, d),( \pm e, b, m),(\mp e, b, m)$, $(\mp e,-b, m),( \pm e,-b, m)$, where $d=m(v-1)$ and $e=\sqrt{h^{2}\left(\frac{2}{v}-1\right)-b^{2}}$, such that $H$ and $\Gamma$ have a common line of symmetry and for a point $P$ in the space the following two statements are equivalent:

(a) A point $P$ is on the ellipsoid $\Gamma$. 
(b) The sum $\left|A C_{P}\right|^{2}+\left|B D_{P}\right|^{2}+\left|C A_{P}\right|^{2}+\left|D B_{P}\right|^{2}$ is equal to the product $v\left(|A C|^{2}+|B D|^{2}\right)$, where $A_{P}, B_{P}, C_{P}$ and $D_{d}$ are the intersections of the plane $A B C D$ with the lines $A^{\prime} P, B^{\prime}, C^{\prime} P$ and $D^{\prime} P$.

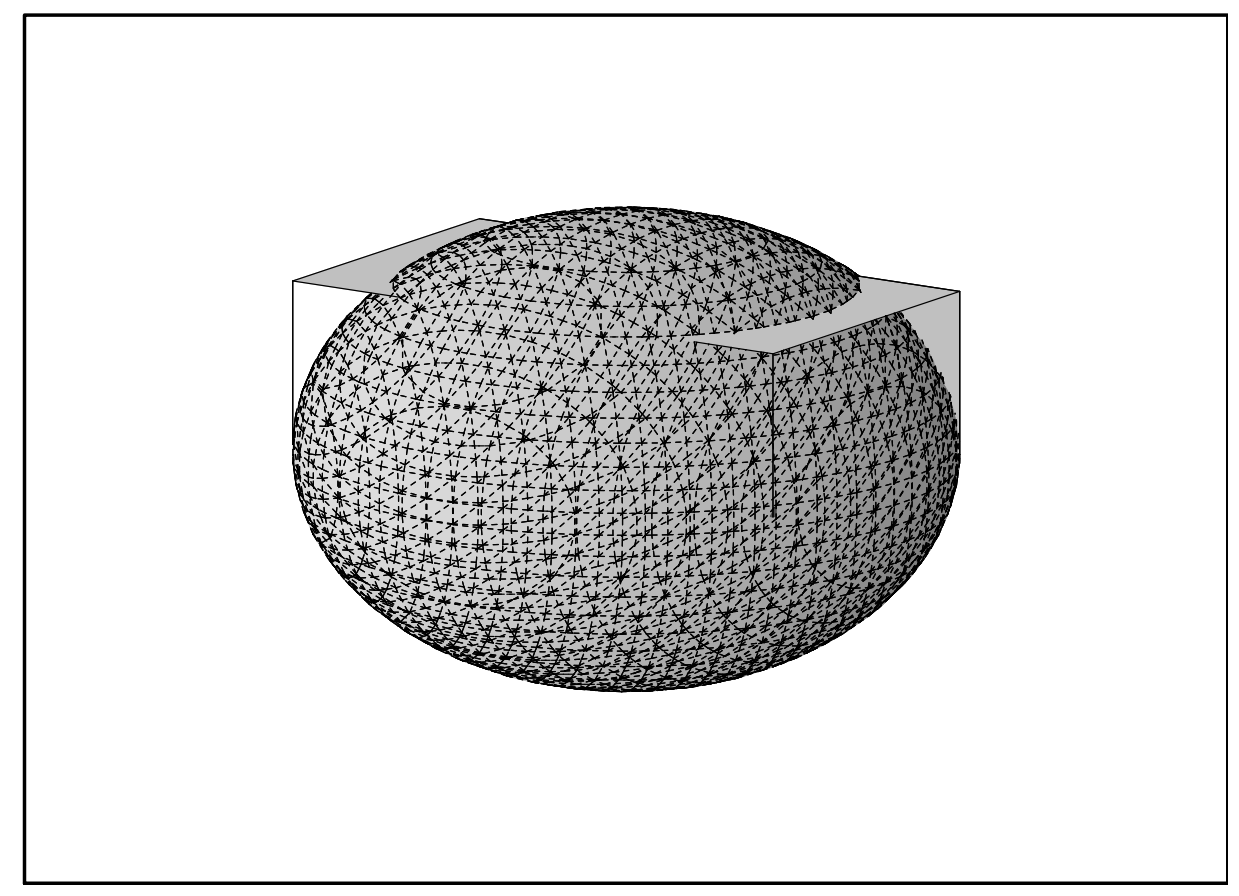

Figure 4: Theorem 3 for $h=1, b=\frac{1}{2}, v=1$ and $m=2$.

Proof. Without loss of generality, we can assume that the above line of symmetry is the $z$-axis of the rectangular coordinate system in the 3 -dimensional Euclidean space and that the coordinates of the vertices $A, B, C, D, A^{\prime}, B^{\prime}$, $C^{\prime}$ and $D^{\prime}$ of a rectangular hexahedron $H$ are $(-a, b, n),(a, b, n),(a,-b n)$, $(-a,-b, n),(-a, b, m),(a, b, m),(a,-b m)$ and $(-a,-b, m)$, where $a, b, n$ and $m$ are real numbers such that $m \neq n, a \neq 0$ and $b \neq 0$. An arbitrary point $P$ in the space has coordinates $(x, y, z)$. Let $\alpha=n-m, \beta=n-z$ and $\gamma=z-m$. The coordinates of the intersections $A_{P}, B_{P}, C_{P}$ and $D_{P}$ have been computed above. Then

$$
\left|A C_{P}\right|^{2}+\left|B D_{P}\right|^{2}+\left|C A_{P}\right|^{2}+\left|D B_{P}\right|^{2}-v\left(|A C|^{2}+|B D|^{2}\right)=\frac{4 \alpha M_{e}}{\gamma^{2}},
$$


where $M_{e}$ is the following quadratic polynomial in $x, y$ and $z$ :

$$
\alpha^{2}\left(x^{2}+y^{2}\right)+c^{2}\left[2(2-v) z^{2}+4(d-n) z-2 v m^{2}+(m+n)^{2}\right] .
$$

Now, in order that $P$ is on the ellipsoid $\Gamma$, the polynomial $M_{e}$ should be of the form $t\left(\frac{x^{2}}{h^{2}}+\frac{y^{2}}{h^{2}}+\frac{z^{2}}{2 v m^{2}}-1\right)$ for some real number $t \neq 0$. This gives the system of ten equations in variables $a, n$ and $t$. The two solutions are $a= \pm e$, $n=d$ and $t=h^{2} m^{2}(v-2)^{2}$ that give the two rectangular hexahedrons $H$ from the statement of the theorem.

Of course, we can apply the above arguments also to the remaining two lines of symmetry of an ellipsoid so that there are three special ellipsoids with two semi-axes equal and the third of the form $m \sqrt{2 v}$ each with two basic rectangular hexahedron $F^{v}$-bases analogous to the ones described above (for the $z$-axis).

The case of a sphere is somewhat special because every line through its center is a line of symmetry for the sphere. By repeating the above proof and solving the system, assuming that the polynomial $M_{e}$ has the form $t\left(\frac{x^{2}}{h^{2}}+\frac{y^{2}}{h^{2}}+\frac{z^{2}}{h^{2}}-1\right)$ for some real number $t \neq 0$, we conclude that for $0<v<2$ and $b<m \sqrt{1-\frac{v}{2}}$ the value $a$ is $\sqrt{\left(1-\frac{v}{2}\right) m^{2}-b^{2}}$ and the radius $h$ of the sphere is $m \sqrt{\frac{v}{2}}$ while $n=d$ as above.

\section{The CASE OF A hyperboloid With tWo SheEts}

When $v>2$, some special hyperboloids with two sheets have rectangular hexahedrons as their $F^{v}$-bases. This is explained in the following result. Once again these rectangular hexahedrons have the $z$-axis as a line of symmetry (see Fig. 5).

Theorem 4. Let real numbers $b, h, m$ and $v$ satisfy $b>0, h>0, m \neq 0$ and $v>2$. Let $\Gamma$ be the hyperboloid with two sheets that has the equation $\frac{z^{2}}{2 v m^{2}}-\frac{x^{2}}{h^{2}}-\frac{y^{2}}{h^{2}}=1$. If $b<h \sqrt{1-\frac{2}{v}}$, then there are two rectangular hexahedrons $H=A B C D A^{\prime} B^{\prime} C^{\prime} D^{\prime}$ with the vertices $( \pm f, b, d),(\mp f, b, d)$, $(\mp f,-b, d),( \pm f,-b, d),( \pm f, b, m),(\mp f, b, m),(\mp f,-b, m),( \pm f,-b, m)$, where $d$ and $f$ are $m(v-1)$ and $\sqrt{h^{2}\left(1-\frac{2}{v}\right)-b^{2}}$, such that $H$ and $\Gamma$ share a line of symmetry and for a point $P$ in the space the following two statements are equivalent:

(a) $A$ point $P$ is on the hyperboloid $\Gamma$.

(b) The sum $\left|A C_{P}\right|^{2}+\left|B D_{P}\right|^{2}+\left|C A_{P}\right|^{2}+\left|D B_{P}\right|^{2}$ is equal to the product $v\left(|A C|^{2}+|B D|^{2}\right)$, where $A_{P}, B_{P}, C_{P}$ and $D_{d}$ are the intersections of the plane $A B C D$ with the lines $A^{\prime} P, B^{\prime}, C^{\prime} P$ and $D^{\prime} P$. 


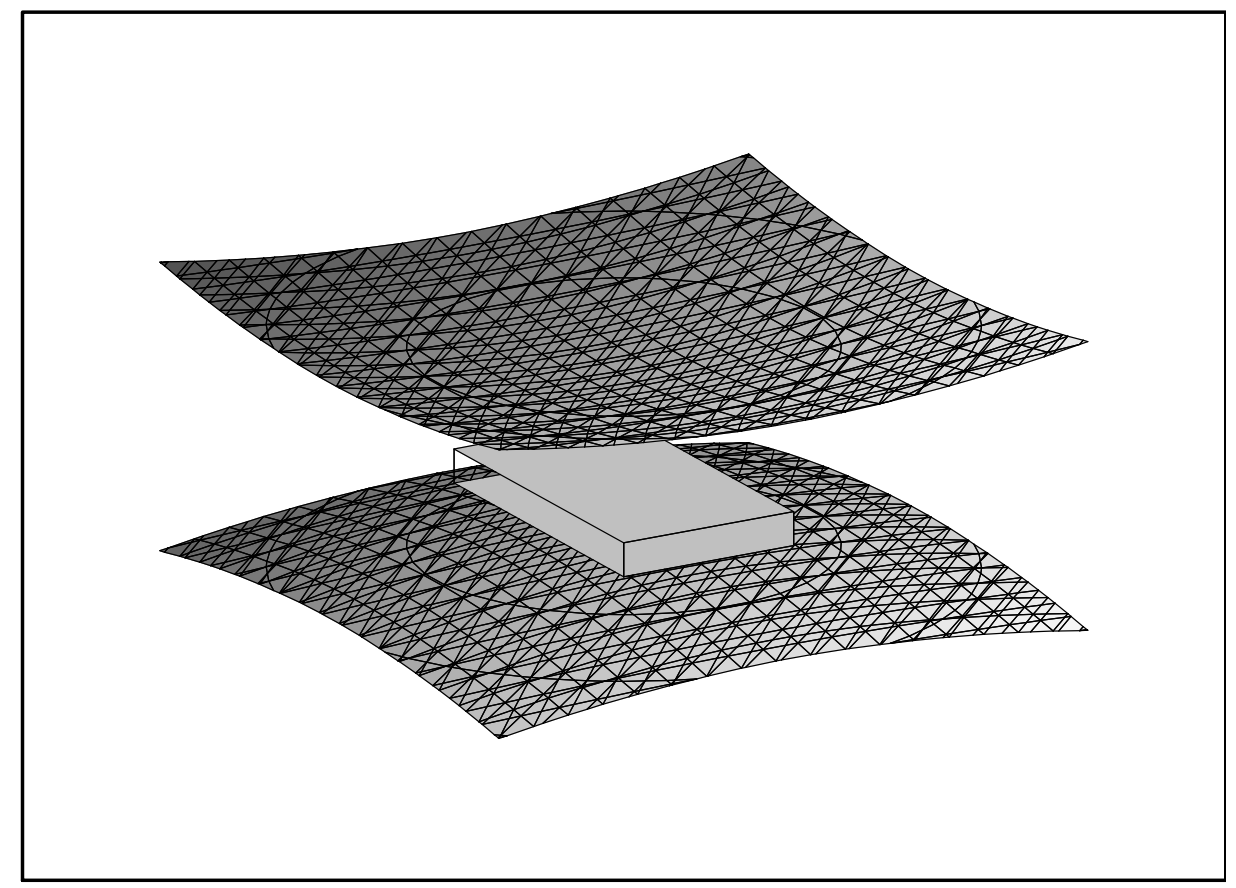

Figure 5: Theorem 4 for $h=1, b=\frac{1}{2}, v=3$ and $m=2$.

Proof. We can repeat the above proof for the ellipsoid and require that the polynomial $M_{e}$ is of the form $t\left(\frac{z^{2}}{2 v m^{2}}-\frac{x^{2}}{h^{2}}-\frac{y^{2}}{h^{2}}-1\right)$ for some real number $t \neq 0$. This gives the system of ten equations in variables $a, n$ and $t$. The two solutions are $a= \pm f, n=d$ and $t=-h^{2} m^{2}(v-2)^{2}$ that give the two rectangular hexahedrons $H$ from the statement of the theorem.

\section{Conclusion}

This note considers the 3-dimensional versions of some recent results by the author and G. M. Gianella that have their origin in an old geometric problem of Fermat in the plane for semicircles and rectangles. The first step of generalization was to replace semicircles with arbitrary conics. In the space rectangles are replaced by rectangular hexahedrons and conics by three kinds of quadrics. As in the plane, this results are proved using methods from analytic geometry. Of course, it remains to deal with arbitrary quadrics perhaps with some other solids. 


\section{REFERENCES}

[1] E. C. Catalan, Théorèmes et problèmes de Géométrie 6e ed., Paris, 1879.

[2] Z. Čerin, On the Fermat geometric problem, Forum Geom., 13 (2013), 135-147.

[3] Z. Čerin, On the modified Fermat problem, Missouri J. Math. Sci., (to appear).

[4] Z. Čerin, Fermat bases of conics, J. Geom., (to appear).

[5] Z. Čerin, Generalization of a geometry problem posed by Fermat, J. Geom. Graph., 17 (2013), 1-5.

[6] Z. Cerin and G. M. Gianella, On the Fermat problem for ellipse, Far East J. Math. Sci., 64 (2012), 213-227.

[7] Leonard Euler, Various Geometric Demonstrations, New Commentaries of the Petropolitan Academy of Sciences I, (1747/48), 1750, pp. 49-66 (English translation prepared in 2005 by Adam Glover).

[8] F. G. -M., Exercices de Géométrie (6e ed.), Éditions Jacques Gabay, Paris 1991. (Reprint of the 6th edition published by Mame and De Gigord, Paris 1920.)

[9] M. L. Hacken, Sur un théorème de Fermat, Mathesis, 27 (1907), 181, 264.

[10] G. A. Korn and T. M. Korn, Mathematical Handbook, McGraw-Hill, New York 1961.

[11] Eugène Lionnet, Solutions des questions proposées, Nouvelles Annales de Mathématiques, Series 2, Vol 9 (1870), pp. 189-191. (This can be downloaded from www. numdam.org)

[12] Ed Sandifer, A forgotten Fermat problem, How Euler Did It, MAA, Washington, DC, 2007.

(Received: June 15, 2012)

(Revised: December 17, 2012)
Kopernikova 7

10010 Zagreb

Croatia

cerin@math.hr 